\title{
Design of a Low-Profile, High-Gain Fabry-Perot Cavity Antenna for $\mathrm{Ku}$-Band Applications
}

\author{
Truong Khang Nguyen ${ }^{1,2,3} \cdot$ Ikmo Park $^{2, *}$
}

\begin{abstract}
A Fabry-Perot resonator cavity antenna for $K u$-band application is presented in this paper. The Fabry-Perot cavity is formed by a ground plane and a frequency selective surface (FSS) made of a circular hole array. The cavity resonance is excited by a single-feed microstrip patch located inside the cavity. The measured results show that the proposed antenna has an impedance bandwidth of approximately $13 \%$ (VSWR $\leq 2$ ) and a $3-\mathrm{dB}$ gain bandwidth of approximately 7\%. The antenna produces a maximum gain of $18.5 \mathrm{dBi}$ and good radiation patterns over the entire $3-\mathrm{dB}$ gain bandwidth. The antenna's very thin profile, high directivity, and single excitation feed make it promising for use in wireless and satellite communication applications in a $K u$-band frequency.
\end{abstract}

Key Words: Directive Antenna, Fabry-Perot Resonator, Frequency Selective Surface.

\section{INTRODUCTION}

The pioneering work of Trentini [1] and further research by James et al. [2] stimulated extensive study on the technique of producing a high-gain planar antenna using a simple source placed between the ground plane and a partially reflective surface (PRS). This technique has overcome several difficulties related to the narrow bandwidth and low antenna efficiency of patch array antennas, particularly when implemented at millimeterwave frequencies $[3,4]$. Applications of this type of antenna, including high-speed wireless local area networks, satellite reception, and various point-to-point radio links, have attracted much interest in recent years with regard to use in the upper microwave, millimeter-wave, and even terahertz regions [5-12].

The operating principle of this type of antenna can be explained in two ways: it is due to the resonant response of the Fabry-Perot-like cavity formed by the PRS and the ground plane, or it is due to the generation of a leaky wave. The gain and bandwidth depend on the reflection (amplitude and phase) from the PRS, as well as the distance from the ground plane. When the patch dipole is combined with the frequency selective surface (FSS), the input impedance of the patch dipole is changed and the resonance frequency of the composite cavity antenna is lowered. Achieving the best possible performance in terms of maximum gain, impedance and gain bandwidth requires careful frequency tuning of the composite cavity antenna. An exhaustive overview of the theory, design, and practical applications of these cavity-type antennas can be found in [13]. A recent review has described the research progress of FabryPerot resonator antennas based on different viewpoints and models of analysis [14], but few papers have focused on optimizing the common bandwidth, i.e., the overlapping frequency range between the impedance bandwidth and the 3-dB gain bandwidth, in a Fabry-Perot cavity resonator antenna. So-

Manuscript received July 14, 2014 ; Revised August 27, 2014 ; Accepted September 4, 2014. (ID No. 20140714-032J)

${ }^{1}$ Division of Energy Systems Research, Ajou University, Suwon, Korea.

${ }^{2}$ Department of Electrical and Computer Engineering, Ajou University, Suwon, Korea.

${ }^{3}$ Faculty of Electronics and Telecommunications, University of Science, Ho Chi Minh, Viet Nam.

*Corresponding Author: Ikmo Park (e-mail: ipark@ajou.ac.kr)

This is an Open-Access article distributed under the terms of the Creative Commons Attribution Non-Commercial License (http://creativecommons.org/licenses/ by-nc/3.0) which permits unrestricted non-commercial use, distribution, and reproduction in any medium, provided the original work is properly cited.

(c) Copyright The Korean Institute of Electromagnetic Engineering and Science. All Rights Reserved. 
me papers obtained broad impedance bandwidth and broad 3$\mathrm{dB}$ gain bandwidth separately. However, due to the mismatch of these frequency ranges, the common bandwidth effectively became narrower.

In this paper, we present the design of a cavity antenna with a broad common bandwidth that uses a Fabry-Perot resonance made of a ground plane and a thin PRS consisting of a twodimensional circular hole array. The antenna structure is designed for the $K u$-band North America uplink (14.0-14.5 GHz) with a maximum gain of about $20 \mathrm{dBi}$. First, we describe the actual prototype of the antenna. The approximate cavity model is then employed to determine the antenna's resonance frequency. Some parameters that significantly affect the antenna characteristics are presented and discussed. Finally, experimental results are shown to validate the design and analysis.

\section{DESCRIPTION AND MODELING}

\section{OFTHE STRUCTURE}

Fig. 1 shows the geometry of the proposed Fabry-Perot cavity antenna. The antenna can be considered as a parallelepipedal flat structure with a square base with $12 \mathrm{~cm}$ sides, which is approximately $5.8 \lambda_{0}$ at $14.4 \mathrm{GHz}$ where $\lambda_{o}$ is free space wavelength. The cavity is bounded by a ground plane and a PRS, where the ground plane acts as a perfect mirror and the circular hole array acts as a partially reflecting mirror. The cavity is filled with a foam whose permittivity is close to one; this ensures the spacing between these two mirrors. The thickness of the foam or the cavity height is denoted as $H$.

The PRS is realized with a circular hole array made of $13 \times$ 13 elementary cells. The circular hole has the advantages of simplicity, unpolarized sensitivity, and increased degree of accuracy in lithography process, especially at high frequencies. In addition, this inductive FSS allows to design on a very thin and low permittivity substrate as a dielectric-free FSS which reduces either the dielectric substrate effects or the cost of a mask market production, particularly in the space applications [15]. The circular hole array is a bi-periodic array, where the periodicity and hole diameter are designated as $P$ and $D$, respectively. The radiating element is a rectangular patch with a width and a length of $W_{p}$ and $L_{p}$, respectively. The patch is placed inside the cavity with a distance of $h_{p}$ above the ground plane. The patch is fed from a coaxial cable coming from the ground plane. The feeding point is off-center along the $x$-axis with a distance of $x_{f}$ to obtain the impedance matching condition and to ensure that the current is predominantly along this axis. The width and the length of the substrate supporting the feeding patch are $A$ and $B$, respectively. Both the circular hole array and the feeding patch are lithographically patterned

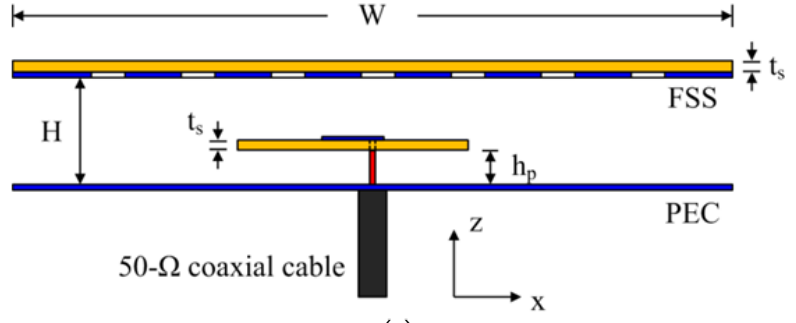

(a)

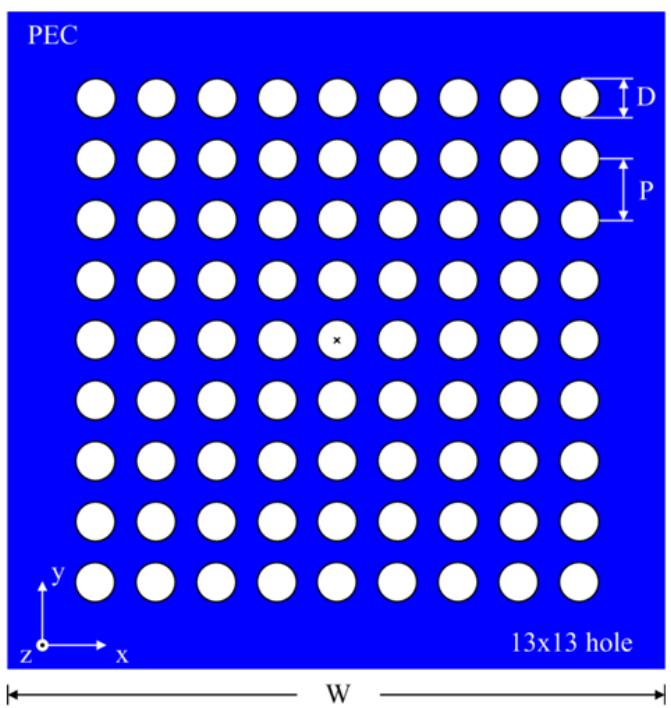

(b)

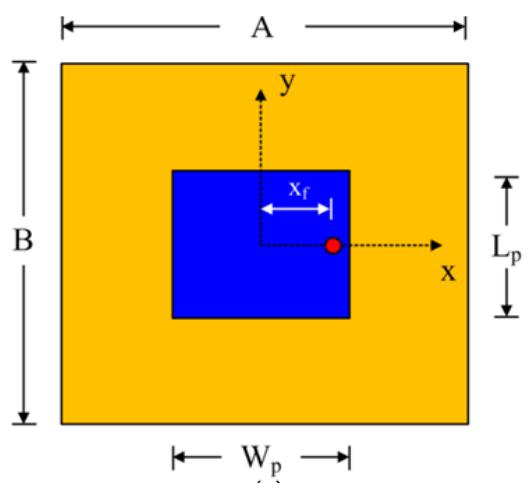

(c)

Fig. 1. Geometry of the proposed antenna with (a) front view, (b) top view of the FSS, and (c) top view of the feeding patch. FSS = frequency selective surface, $\mathrm{PEC}=$ perfect electric conductor.

on the same Duroid RO4003 substrate, whose relative permittivity and thickness are 3.38 and $0.508 \mathrm{~mm}$, respectively. The design parameters for optimum antenna performance are summarized in Table 1.

An electromagnetic simulation was performed using CST Microwave Studio (CST MWS; Computer Simulation Technology AG., Darmstadt, Germany) to analyze the characteristics of the proposed antenna. The transmission characteristics were first studied for a unit cell model consisting of a half-wavelength resonator, a single circular hole supported by RO4003 substrate, and a ground plane. The structure was 
Table 1. Design parameters of the proposed antenna for optimum performance

\begin{tabular}{cccc}
\hline Parameter & $\begin{array}{c}\text { Dimension } \\
(\mathrm{mm})\end{array}$ & Parameter & $\begin{array}{c}\text { Dimension } \\
(\mathrm{mm})\end{array}$ \\
\hline$W$ & 120 & $A$ & 46 \\
$H$ & 9.4 & $B$ & 45 \\
$h_{p}$ & 1.7 & $W_{p}$ & 6 \\
$t_{s}$ & 0.508 & $L_{p}$ & 5 \\
$P$ & 8.3 & $x_{f}$ & 2 \\
$D$ & 6.4 & & \\
\hline
\end{tabular}

modeled as an infinite array in the CST MWS by utilizing CST's full Floquet mode implementation to simulate a single unit cell of the FSS. The periodic unit cell boundary condition is applied, which virtually repeats the modeled structure periodically and infinitely in two directions. The transmission behavior and the resonance frequency can be easily predicted by this simplified model, which is excited by a normal incident plane wave. The reason is that the beamwidth of the antenna is

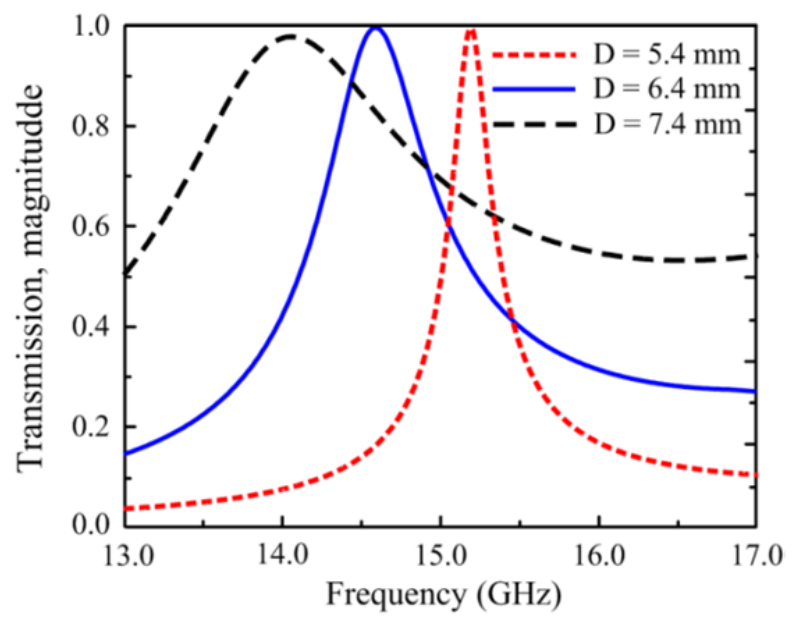

(a)

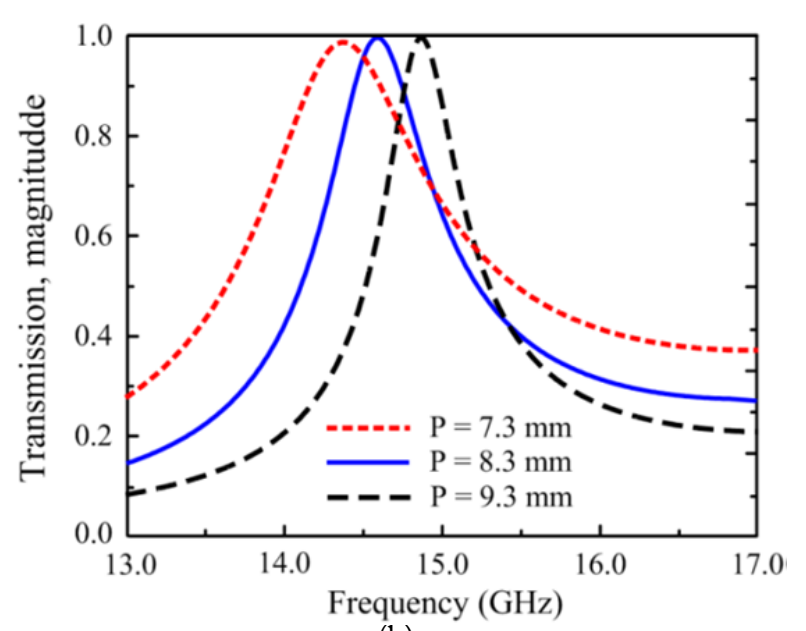

(b)

Fig. 2. Transmission characteristics of the unit cell structure as a function of (a) hole diameter and (b) hole array periodicity.

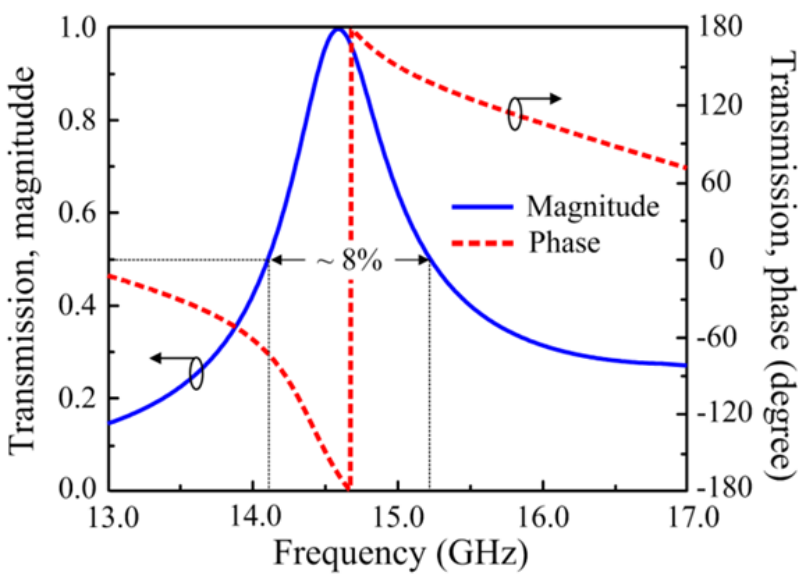

Fig. 3. Simulated transmission coefficient of the cavity using a unit cell model, for normal incidence.

directly linked with the angular selectivity of the structure illuminated by a plane wave under the incident angle $\theta$ [7]. In this way, the directivity of the Fabry-Perot cavity type antenna related to the primary source in the bore-sight direction $\left(\theta=0^{\circ}\right)$ can be estimated at a certain frequency.

Fig. 2 shows the transmission of the cavity as a function of the hole diameter and hole array periodicity. The resonant frequency and the bandwidth of the unit cell are controlled by the hole diameter and periodicity. The larger hole diameter resulted in a decrease in the resonance frequency while the larger periodicity resulted in an increase in the resonance frequency. Wider transmission bandwidths can be obtained at the lower frequencies; however, the transmission magnitude is reduced. Fig. 3 shows the transmission magnitude and phase of the optimized unit cell. This result indicates that transmission is maximum at a resonant frequency and the resonant frequency is near the frequency of $180^{\circ}$ transmission phase, i.e., $14.6 \mathrm{GHz}$ in the present case. The proposed FSS-patch composite antenna can be used in the present North America $K u$-band very small aperture terminal for satellite communications, which demands a transmission frequency of 14-14.5 GHz.

After optimizing the parameter of the unit cell and the distance between the FSS and ground plane, the predicted transmission bandwidth at half-maximum is approximately $8 \%$, i.e., from 14.1 to $15.2 \mathrm{GHz}$. Therefore, the proposed antenna has satisfied the transmission bandwidth requirement. In addition, the required minimum gain of about $20 \mathrm{dBi}$ has also been satisfied owning to the low quality factor (Q) of the FSS screen.

\section{ANTENNA CHARACTERISTICS}

After optimization of the transmission characteristic of a resonator based on the unit cell model, a FSS structure was 


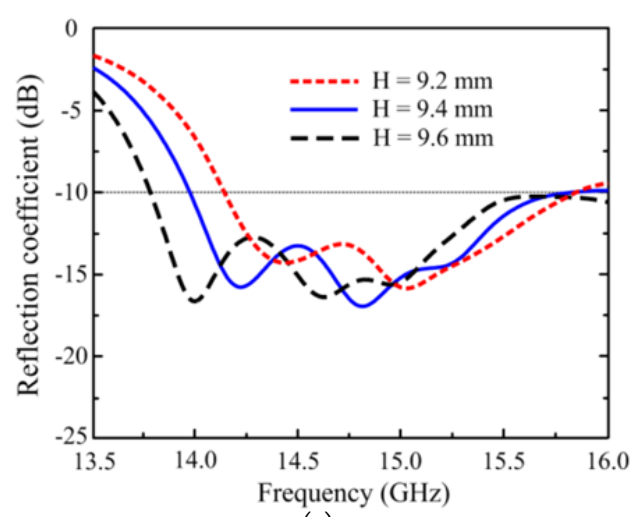

(a)

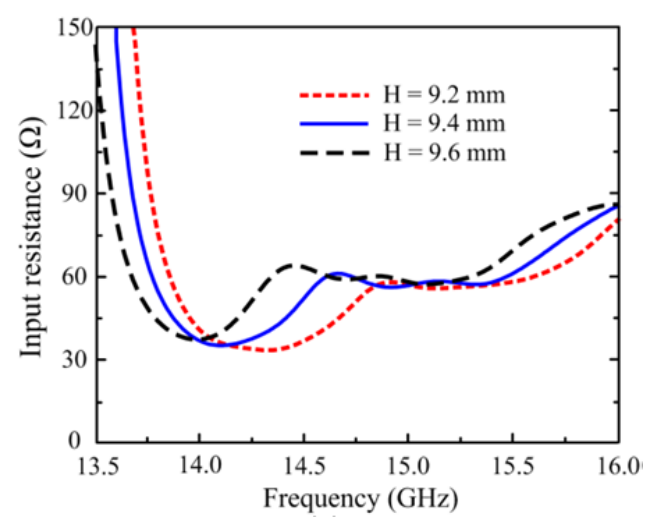

(b)

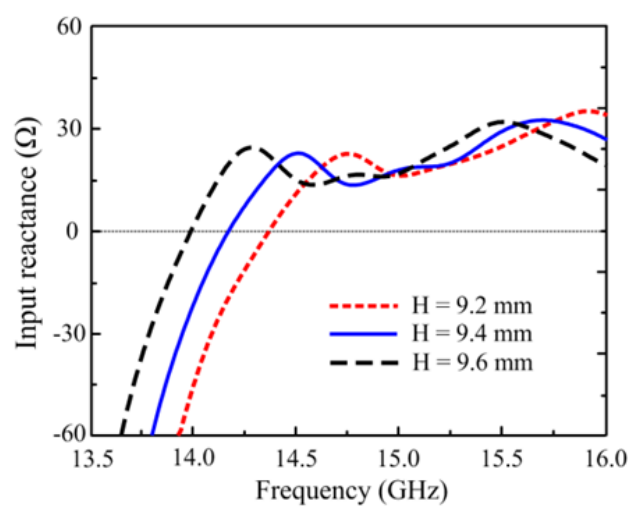

(c)

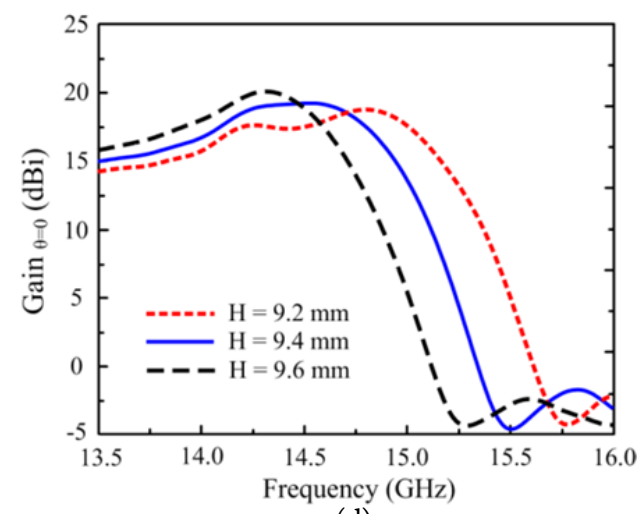

(d)

Fig. 4. Effect of the cavity height, $H$, on antenna characteristics with respect to (a) the reflection coefficient, (b) input resistance, (c) input reactance, and (d) broadside gain. designed on top of the patch and the ground plane. The reflection coefficient, input impedance, and gain of the antenna at resonance were studied and analyzed. In the design, two parameters-the distance, $H$, between the ground plane and the FSS, and the location of the patch inside the cavity, $h_{p}$-were chosen to study the reflection coefficient and gain characteristics of the antenna.

Fig. 4 shows the antenna characteristics with respect to the cavity height $H$. The cavity height significantly affected the antenna characteristics in terms of both the reflection coefficient and gain. We obtained two main resonances in both the reflection coefficient and gain responses. Note that the resonances in the reflection coefficient correspond to the local minima while the resonances in the gain correspond to the local maxima. When the cavity height was increased from 9.2 to 9.6 $\mathrm{mm}$ in $0.2 \mathrm{~mm}$ steps, two resonances in the reflection coefficient linearly shifted to the lower frequency in a simultaneous manner. One addition local minimum was introduced and became more distinct for the thicker cavity, e.g., $H=9.6$ $\mathrm{mm}$. These behaviors can be explained based on the resonant loading effect of the FSS superstrate. The impedance characteristic shows that the primary resonance in the antenna's impedance occurred in the low frequency region where the antenna input impedance had zero reactance. However, only the upper resonance in the gain response changed with respect to the cavity height variation. This indicates that the primary resonance in the antenna gain is determined by the resonance length of the cavity, and occurs at the upper frequency region. In conjunction with the impedance characteristics of the antenna, this demonstrates the difficulty of matching these two frequency ranges in a composite high-gain cavity antenna. The upper resonance in the antenna gain decreased with the increase in cavity height. As the cavity height increased from 9.2 to 9.4 to $9.6 \mathrm{~mm}$, the upper resonance frequency decreased from 14.8 to 14.6 to $14.4 \mathrm{GHz}$, respectively. Nevertheless, the lower resonance frequency remained around $14.2 \mathrm{GHz}$. For the thicker cavity of $9.6 \mathrm{~mm}$, the maximum gain exhibited an increase; however, the two resonances merged together and this reduced the gain bandwidth of the antenna. The lower cavity height, that is, $H=9.2 \mathrm{~mm}$, produced a wider gain bandwidth, but the fractional impedance bandwidth became mismatched with the $3-\mathrm{dB}$ gain bandwidth of the antenna. As a result, as a general consideration of antenna performance, the common bandwidth, which is the overlapping frequency range between the impedance bandwidth and the 3-dB gain bandwidth, effectively became narrower. Therefore, the optimized cavity height was chosen as $9.4 \mathrm{~mm}$ for the best possible common bandwidth and maximum gain. The impedance and gain bandwidths of the antenna for different $H$ values are summarized in Table 2. 
Table 2. Impedance and gain bandwidths of the antenna for different $H$ values

\begin{tabular}{cccc}
\hline Parameter & $\begin{array}{c}\text { Impedance bandwidth } \\
\left(S_{11} \leq-10 \mathrm{~dB}\right)(\mathrm{GHz})\end{array}$ & $\begin{array}{c}\text { Gain bandwidth } \\
\left(\mathrm{G}_{\max } \pm 3 \mathrm{dBi}\right)(\mathrm{GHz})\end{array}$ & $\begin{array}{c}\text { Common bandwidth } \\
(\mathrm{GHz})\end{array}$ \\
\hline$H=9.2 \mathrm{~mm}$ & $14.14-15.85(11.4 \%)$ & $14.00-15.13(7.7 \%)$ & $14.14-15.13(6.7 \%)$ \\
$H=9.4 \mathrm{~mm}$ & $13.91-15.86(12.7 \%)$ & $13.90-14.91(7.0 \%)$ & $13.91-14.91(7.0 \%)$ \\
$H=9.6 \mathrm{~mm}$ & $13.78-16.34(17.0 \%)$ & $13.84-14.62(5.3 \%)$ & $13.84-14.62(5.5 \%)$ \\
\hline
\end{tabular}

Fig. 5 presents the reflection coefficient and gain characteristics with respect to the distance between the feeding patch and the ground, or the patch height. As the patch height, $h_{p}$, increased from 1.5 to $1.9 \mathrm{~mm}$ in steps of $0.2 \mathrm{~mm}$, the lower resonance frequency decreased from 14.4 to $14.0 \mathrm{GHz}$ in 0.2 $\mathrm{GHz}$ steps. The higher resonance frequency remained at around $14.8 \mathrm{GHz}$. As the height of the patch increased, so did the length of the feeding pin of the coaxial cable, and consequently, the inductance of the patch. Therefore, the resonance frequency of the feeding pin with a square patch decreased, shifting to a lower frequency. On the other hand, the increases in the patch height produced a stronger coupling between the two resonances. This was attributed to the coupling between the patch and the FSS. Since the total height of the

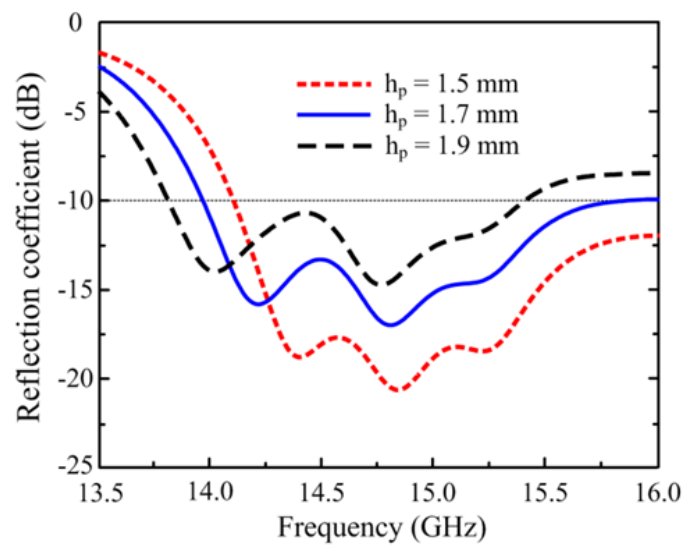

(a)

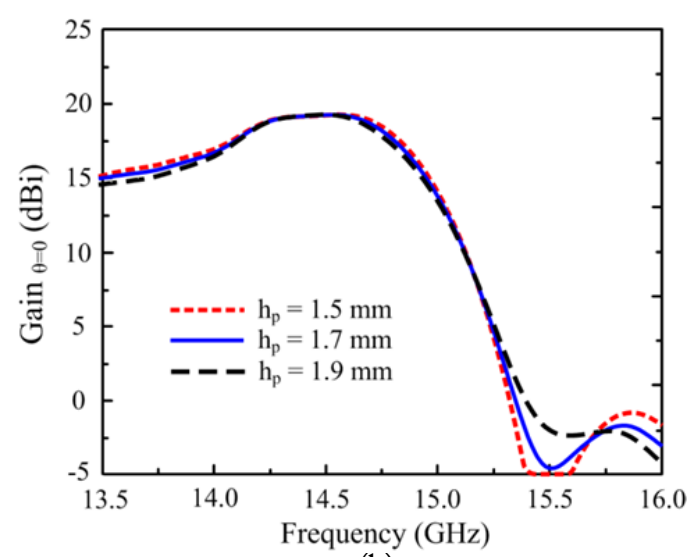

(b)

Fig. 5. Effect of the patch height, $h_{p}$, on antenna characteristics with respect to (a) the reflection coefficient and (b) broadside gain. cavity did not change with changes in the patch height, the lower and higher resonance frequencies in the gain response remained around their original values. These results show that the antenna behavior as a function of the frequency can be manipulated with these design parameters to maximize the common bandwidth of the antenna, so that the impedance bandwidth fully covers the $3-\mathrm{dB}$ gain bandwidth.

\section{RESULTS}

Fig. 6 shows the photographs of the fabricated antenna. The performance of the antenna was measured using an Agilent PNA N5232A network analyzer. Fig. 7 shows that the measured reflection coefficient is close to the simulated one. A measured impedance bandwidth of about $1.9 \mathrm{GHz}(13.9-15.8$ $\mathrm{GHz}$ ) was obtained for $\mathrm{VSWR} \leq 2$, which corresponds to a fractional bandwidth of approximately $13 \%$ for the center frequency of $14.85 \mathrm{GHz}$.

Fig. 8 shows that the measured gain was also close to the simulated one. A measured $3-\mathrm{dB}$ gain bandwidth of about 1.0 $\mathrm{GHz}(13.8-14.8 \mathrm{GHz})$ was obtained, corresponding to approximately $7 \%$ for the center frequency of $14.4 \mathrm{GHz}$. In conjunction with the impedance bandwidth, a common bandwidth of approximately $6.3 \%(13.9-14.8 \mathrm{GHz})$ was achieved for the proposed Fabry-Perot cavity antenna. The measured maximum gain of the proposed antenna was approximately $18.5 \mathrm{dBi}$ at 14.4 $\mathrm{GHz}$, which is about $0.7 \mathrm{~dB}$ lower than the simulated value. In addition, a frequency shift was observed in the high frequency region. These slight discrepancies are attributed to two factors: First, the exact permittivity of the foam is not known. Second, the foam thickness is not perfectly constant and equal to $9.4 \mathrm{~mm}$, as expected in the simulation.

The radiation patterns in the $x z$-plane (E-plane) and in the $y z$-plane (H-plane) at various frequencies (i.e., from 13.4 to 14.6 GHz in increments of $0.4 \mathrm{GHz}$ ) are shown in Fig. 9. The measured results are in agreement with the simulated ones. The radiation patterns in the $x z$-plane exhibited an asymmetry that is attributed to the fact that the excitation point of the feeding patch was off-center and the fabrication tolerance in the realization of the antenna. The antenna exhibited a good radiation pattern characteristic, with clean main lobe and low side lobe levels over the entire the $3-\mathrm{dB}$ gain frequency bandwidth. We 


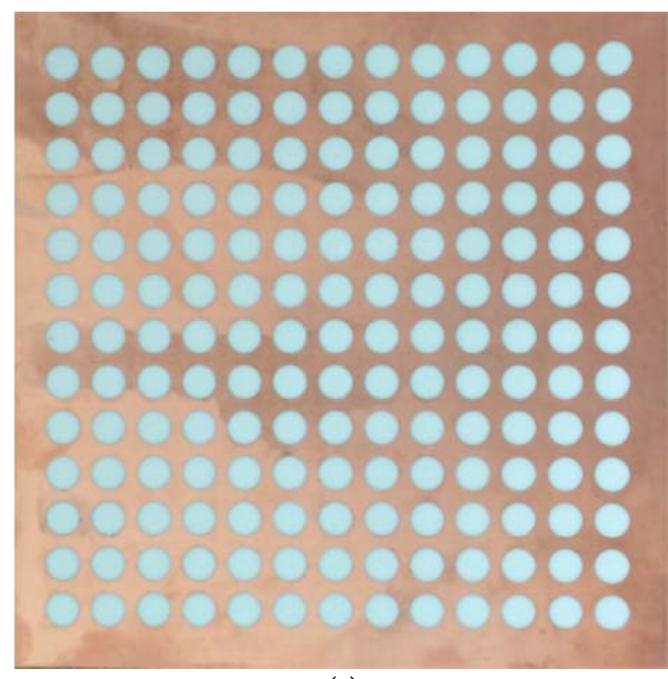

(a)

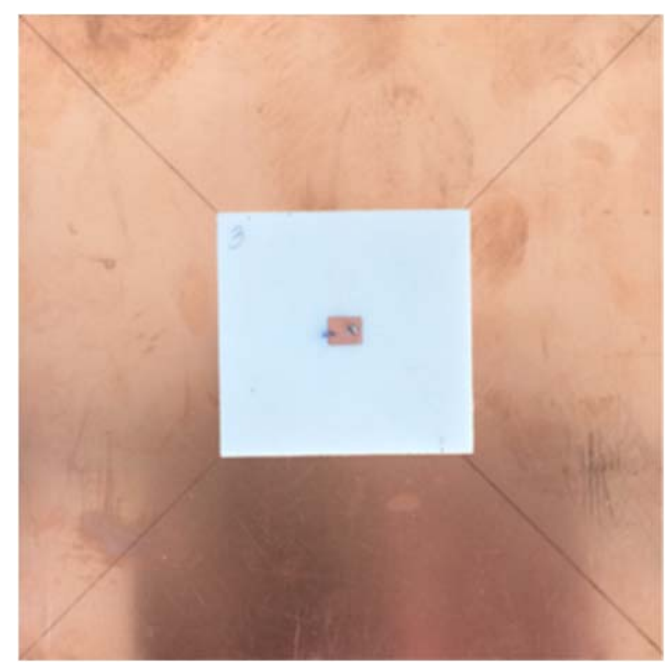

(b)

Fig. 6. Photograph of the fabricated antenna with (a) top view of the FSS and (b) top view of the feeding patch with the ground plane.

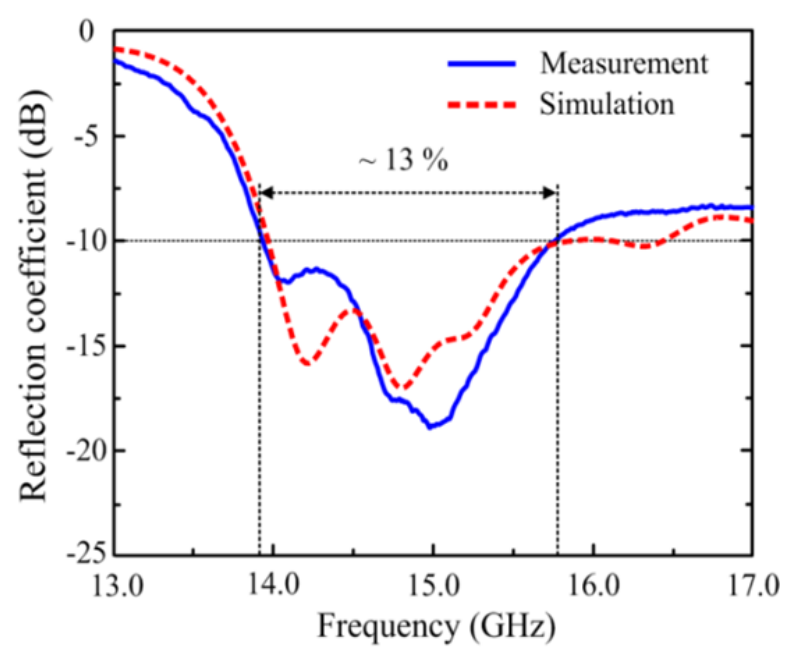

Fig. 7. Reflection coefficient of the proposed antenna in a comparison of simulation and measurement.

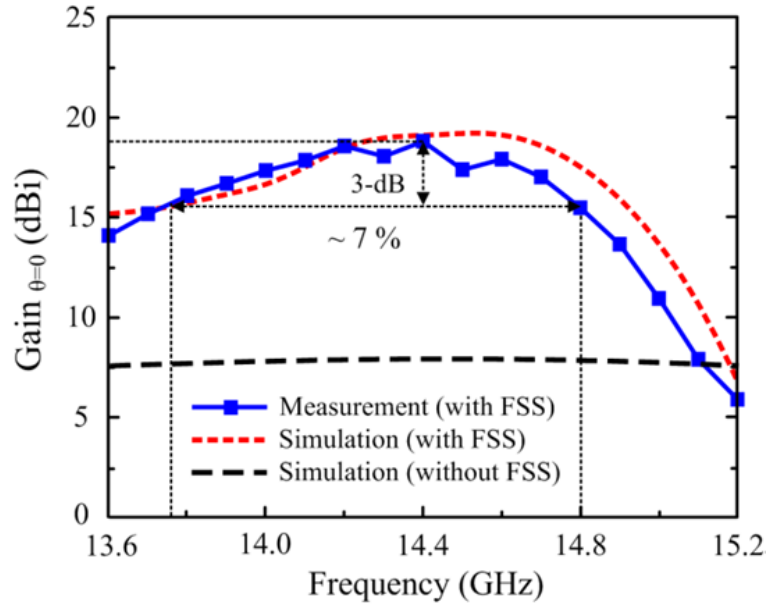

Fig. 8. Simulated and measured broadside gain versus frequency of the proposed antenna.

observed in the simulation that the main beam splitting occurred for frequencies higher than $15.0 \mathrm{GHz}$. For wavelengths $(\lambda)$ shorter than the resonance wavelength $\left(\lambda_{r}\right)$, the transmission maximum was not along the normal direction $(\theta=0)$, but rather along the direction defined by $\theta=a \cos \left(\lambda \lambda_{r}\right)$. On the other hand, for wavelengths $(\lambda)$ longer than the resonance wavelength $\left(\lambda_{r}\right)$, the transmission maximum corresponded to $\theta$ $=0$; thus, the antenna emitted a main lobe focused around the normal of the structure. However, at wavelengths far longer than the resonance wavelength (i.e., frequencies lower than 12.2 $\mathrm{GHz}$ ), the beam along the normal direction deteriorated and most of the radiated power of the antenna contributed to the side and back radiations.

\section{CONCLUSIONS}

We presented a low-profile, broad common bandwidth, high-gain antenna using Fabry-Perot cavity resonance for $\mathrm{Ku}$ band applications at 13.9-14.8 GHz. The results of the simulation and measurement exhibited good agreement. A high gain and broad common bandwidth were obtained (18.5 dBi in maximum gain and $7 \%$ in common bandwidth). In addition to its decreased thickness and the fact that it is a single feed device, the weight of the proposed antenna is low; thus, it could be promising for wireless and satellite communication applications in $\mathrm{Ku}$-band frequencies.

This work was supported by ICT R\&D Program of MSIP/IITP (No. 14-911-01-001). Truong Khang Nguyen would like to thank the Vietnam National Foundation for Science and Technology Development (NAFOSTED) for supporting him under grant (No. 103.05-2013.75) 

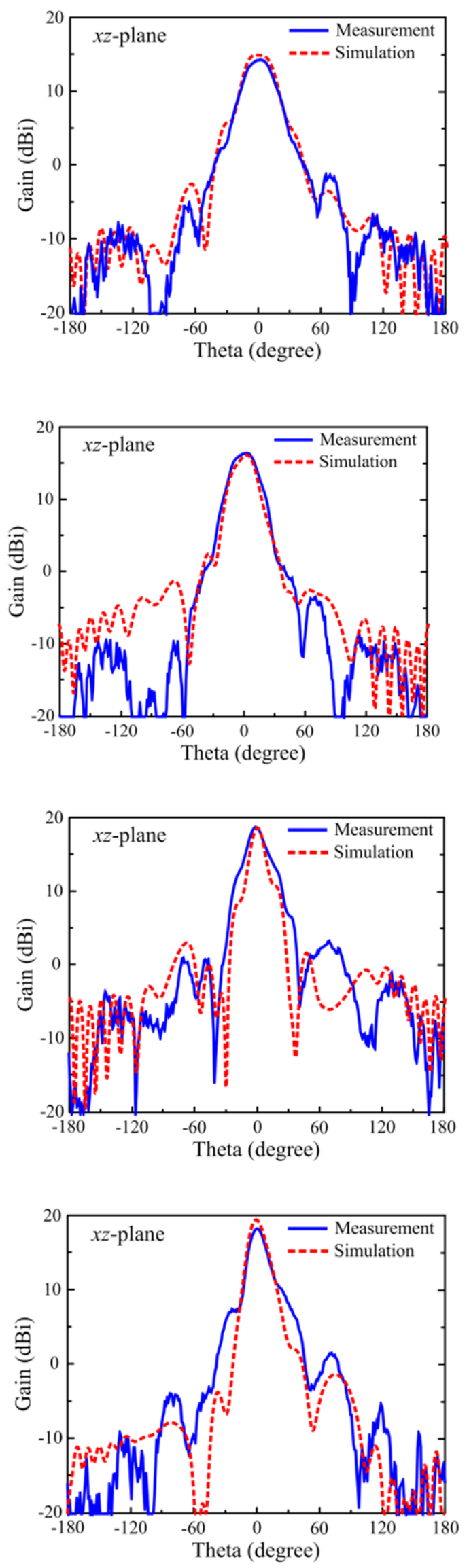

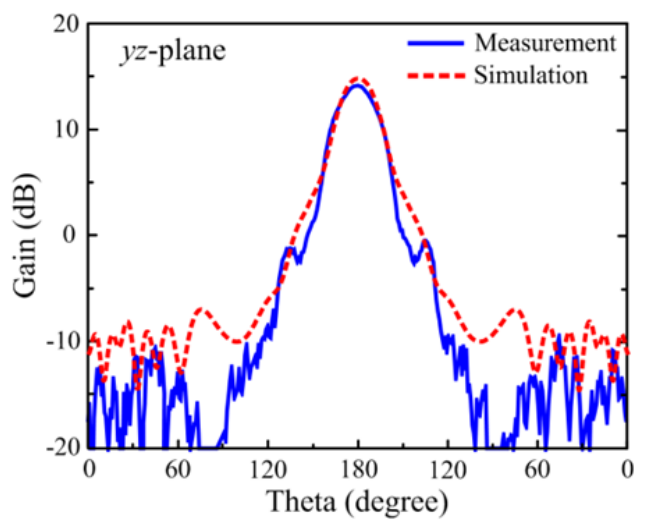

(a)

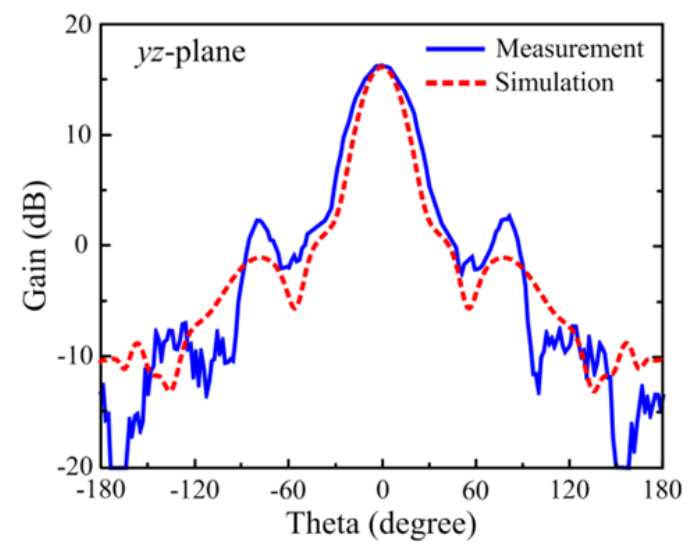

(b)

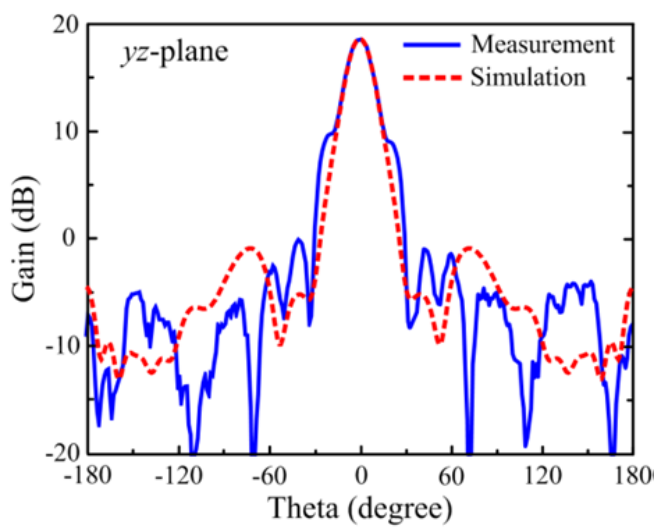

(c)

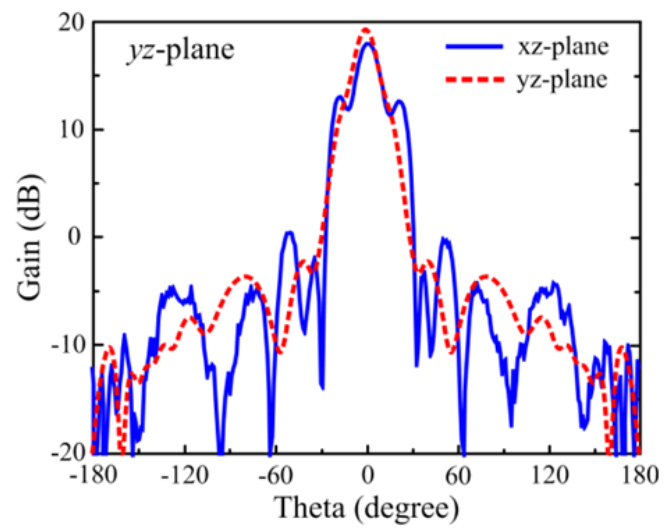

(d)

Fig. 9. Simulated and measured radiation patterns of the proposed antenna in the $x z$ - and $y z$-planes at different frequencies: (a) $13.4 \mathrm{GHz}$, (b) 13.8 $\mathrm{GHz}$, (c) $14.2 \mathrm{GHz}$, and (d) $14.6 \mathrm{GHz}$. 


\section{REFERENCES}

[1] G. V. Trentini, "Partially reflecting sheet arrays," IRE Transactions on Antennas Propagation, vol. 4, no. 4, pp. 666671, 1956.

[2] J. R. James, S. J. A. Kinany, P. D. Peel, and G. Andrasic, "Leaky wave multiple dichroic beamformers," Electronics Letters, vol. 25, no. 18, pp. 1209-1211, 1989.

[3] J. R. James and P. S. Hall, Handbook of Microstrip Antennas. London: Peter Peregrinus, 1989.

[4] D. M. Pozar and D. H. Schaubert, Microstrip Antennas: The Analysis and Design of Microstrip Antennas and Arrays. New York, NY: Institute of Electrical and Electronics Engineers, 1995.

[5] D. Kim and J. Yeo, "A new resonance prediction method of Fabry-Perot cavity (FBC) antennas enclosed with metallic side walls," Journal of the Korean Institute of Electromagnetic Engineering and Science, vol. 11, no. 3, pp. 220-226, Sep. 2011.

[6] T. K. Nguyen and I. Park, "Compact slit antenna incurporated with frequency selective surface," in Global Symposium on Millimeter Waves Digest, Seoul, Korea, 2014, pp. 30-31.

[7] A. P. Feresidis and J. C. Vardaxoglou, "High gain planar antenna using optimised partially reflective surfaces," IEE Proceeding of Microwaves, Antennas and Propagation, vol. 148, no. 6, pp. 345-350, 2001.

[8] N. Guerin, S. Enoch, G. Tayeb, P. Sabouroux, P. Vincent, and H. Legay, "A metallic Fabry-Perot directive antenna," IEEE Transactions on Antennas and Propagation, vol. 54, no.

Truong Khang Nguyen

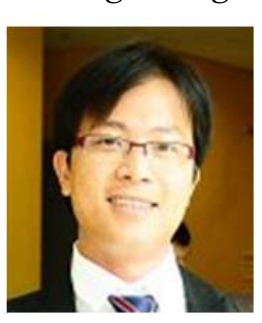

received the B.S. degree in Computational Physics from the University of Science, Vietnam National University, Ho Chi Minh City, and the M.S. and $\mathrm{Ph} . \mathrm{D}$. degrees in Electrical and Computer Engineering from Ajou University in Suwon, Korea. He is currently a Postdoctoral Research Fellow at Ajou University in the Division of Energy Systems Research. $\mathrm{He}$ is also a lecturer at the University of Science, Vietnam National University, Ho Chi Minh City, in the Department of Electronics and Telecommunications. He has authored and co-authored over 40 technical journal and conference papers. He has writen one book chapter in the area of terahertz antenna. His current research interests include the design and analysis of microwave, millimeter-wave, terahertz wave, and nanostructured antennas.
1, pp. 220-224, Jan. 2006.

[9] S. Zvanovec, P. Cerny, P. Piksa, T. Korinek, P. Pechac, M. Mazanek, J. Varga, J. Koubek, and S. Urban, "The use of the Fabry-Perot interferometer for high resolution microwave spectroscopy," Journal of Molecular Spectroscopy, vol. 256, no. 1, pp. 141-145, Jul. 2009.

[10] R. Sauleau, P. Coquet, J. P. Daniel, T. Matsui, and N. Hirose, "Analysis of millimeter-wave Fabry-Perot cavities using the FDTD technique," IEEE Microwave Guided Wave Letters, vol. 9, no. 5, pp. 189-191, 1999.

[11] P. Piksa, S. Zvanovec, and P. Cerny, "Specific millimeterwave features of Fabry-Perot resonator for spectroscopic measurements," in Microwave and Millimeter Wave Technologies from Photonic Bandgap Devices to Antenna and Applications, I. Minin, ed., Rijeka, Croatia: InTech, 2010, pp. 451-468.

[12] T. K. Nguyen, T. A. Ho, I. Park and H. Han, "Full-wavelength dipole antenna on a GaAs membrane covered by a frequency selective surface for a terahertz photomixer," Progress in Electromagnetics Research, vol. 131, pp. 441-455, 2012.

[13] A. Kumar and H. D. Hristov, Microwave Cavity Antennas. Northwood, MA: Artech House, 1989.

[14] Z. G. Liu, "Fabry-Perot resonator antenna," Journal of Infrared, Millimeter, and Terahertz Waves, vol. 31, no. 4, pp. 391-403, 2009.

[15] D. Ramaccia, A. Toscano, A. Colasante, G. Bellaveglia, and R. Lo Forti, "Inductive tri-band double element FSS for space applications," Progress in Electromagnetics Research C, vol. 18, pp. 87-101, 2011.

Ikmo Park

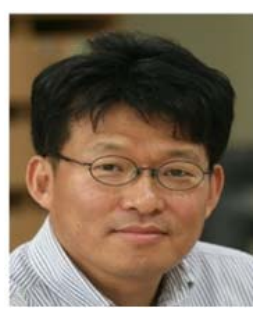

received the B.S. degree in Electrical Engineering from the State University of New York at Stony Brook, and M.S. and Ph.D. degrees in Electrical Engineering from the University of Illinois at Urbana-Champaign. $\mathrm{He}$ joined the Department of Electrical and Computer Engineering at Ajou University in March, 1996. Prior to joining Ajou University, he has been working with the Device \& Materials Laboratory of LG Corporate Institute of Technology, Seoul, Korea, where he had been engaged in research and development of various antennas for personal communication systems, wireless local area networks, and direct broadcasting systems. He was a Visiting Professor with the Department of Electrical and Computer Engineering, POSTECH, Pohang, Korea, from March 2004 to February 2005, and the Department of Electrical and Computer Engineering, University of Arizona, Tucson, Arizona, USA, from July 2011 to June 2012. He has authored and co-authored over 200 technical journal and conference papers. He also holds over 30 patents. 